\title{
Escrituras de mujeres, las huellas del feminismo en las revistas de los Centros Académicos Independientes, Chile 1980-19901
}

\author{
Women's Writings, Traces of Feminism in Journals of \\ the Independent Academic Centers, Chile 1980-1990
}

\begin{abstract}
Resumen
Este artículo revisa la red textual que constituyeron las revistas Proposiciones, Apuntes para el Trabajo Social y el área de documentos de Trabajo de Flacso. Este grupo constituyó parte de los espacios donde las mujeres intelectuales de las ciencias sociales, que realizaron investigación-acción, utilizaron como medio para difundir los debates respecto de las condiciones de vida de las mujeres populares, dialogando con el feminismo y con la izquierda en proceso de renovación.

El objetivo central corresponde a la identificación de los corpus de debates en los participaron las mujeres intelectuales socialistas, junto con resaltar el esfuerzo para generar una intersección entre el género y la clase. Desde 1980 hasta 1990, las revistas tuvieron a las mujeres como un actor central, pese a que disputaron esos espacios con otros intelectuales de la época. Así lo más valioso de estas escrituras es su carácter abierto al debate en las ciencias sociales, donde se instalan las marcas de un feminismo que aspiraba a dotar de sentido a lo popular.
\end{abstract}

Palabras claves

Mujeres, Revistas Político-académicas, ONG, feminismo popular.

1 Este artículo fue realizado gracias al financiamiento del Proyecto Posdoctorado DICYT 031552MB de la Universidad de Santiago de Chile y al Proyecto Fondecyt 1150049 de CONICYT. 


\begin{abstract}
This article reviews the textual network that constituted the publications "Proposiciones", "Apuntes para el trabajo social" and the area of "documentos de trabajo" of Flacso. This group was part of the spaces where the intellectual women of the social sciences, who carried out action research, used as a means to disseminate the debates regarding the living conditions of the popular women, dialoguing with feminism and with the left in process of renewal.

The central objective corresponds to the identification of the corpus of debates in the participation of the socialist intellectual women, together with highlighting the effort to generate an intersection between gender and class. From 1980 to 1990, the reviews had women as a central actor, although they disputed those spaces with other intellectuals of the time. Thus the most valuable of these writings is its openness to debate in the social sciences, where the brands of a feminism that aspired to give meaning to the popular are installed.
\end{abstract}

Keywords

Women, Political-academic journals, NGO, popular feminism.

Narrar y escribir la experiencia social femenina ha sido parte de la lucha de las mujeres por visibilizar sus condiciones de subalternidad, de explotación y de exclusión. Tal como plantea Ernesto Laclau, "algo requiere ser narrado en la medida en que su especificidad escapa a una determinación teórica directa, a un complejo institucional autorreferencial” (Arfurch 11). En América Latina y en Chile en particular, los años ochenta fueron el escenario de la reemergencia de las escrituras femeninas. En forma de boletines, de pasquines, pero también en revistas académicas y políticas, las mujeres plasmaron sus experiencias y problematizaron su realidad.

La denominada segunda oleada feminista, ocupó un lugar protagónico en la lucha contra la dictadura imprimiendo no sólo una narrativa de resistencia y oposición, sino que disputando los sentidos del horizonte democrático. En este artículo nos centraremos en la recuperación de las escrituras que hicieron mujeres intelectuales de oposición, en particular aquellas que desde las ciencias sociales produjeron saberes nacidos al alero de prácticas de intervención social, tensionando los cánones discursivos de la disciplina sociológica y del trabajo social, para instalar un conjunto de debates respecto del género y la clase. Tal como definió Raquel 
Olea, mujeres profesionales e intelectuales, transformaron el saber experto en un instrumento de acción política, haciendo de:

[...] el lugar de las prácticas y las experiencias de las mujeres una narración que produce saberes [...] la experiencia como relato de apertura a otro saber. El conocimiento feminista se ha producido relacionado a las experiencias particulares de las mujeres, en la producción de otredad no nombrada previamente por lo dominante. La experiencia no es expropiada -sino parte- de la productividad teórica. (Olea 251)

El espacio de circulación de estos debates se dio en el nuevo campo intelectual que se construyó durante la dictadura militar. ONGs y Centros Académicos Independientes reemplazaron a las universidades, cerrada para estas investigaciones y, en general, para todas las ciencias sociales acusadas por la Junta Militar de promover la ideología marxista y la radicalización política. Mayoritariamente financiadas con fondos provenientes de fundaciones que favorecieron la renovación del pensamiento socialista y el fortalecimiento de la sociedad civil, mujeres profesionales hicieron de la práctica de intervención en el espacio social popular, un lugar para reflexionar sobre la condición de la mujer en la intersección del género y la clase.

Las condiciones materiales de producción de los saberes, mediante proyectos que recibían generalmente menos recursos que los de sus pares masculinos, estuvieron marcadas por cierto menosprecio de su calidad académica y el valor de las reflexiones. Aunque constituyeron parte de un círculo más amplio de intelectuales, formados en ciencias sociales y con trayectorias militantes en la izquierda socialista, configuraron un grupo distinguible por hacer de los debates sobre la mujer un tema y una agenda que posibilitó recepcionar en Chile un conjunto de reflexiones transnacionales que habitaban el heterogéneo mundo del feminismo de la segunda oleada. 
La disputa por la democracia, la lucha por los derechos humanos y el trabajo de campo en la intervención social que realizaron en poblaciones populares, situadas en las zonas sur, oriente y norte de Santiago, articularon un campo de saber diferenciado, que ganó un estatus reflexivo en el conocimiento social de los años 80. Aquí recuperamos esas escrituras femeninas, poniendo especial atención a aquellas que se formalizaron en la importante revista Proposiciones de la ONG SUR, Apuntes para el trabajo social del colectivo de Trabajo Social y en Flacso, espacios que constituyeron parte de una red más amplia de generación de saberes sobre lo social popular y lo político, insertas dentro del complejo y heterogéneo proceso de renovación socialista.

En particular, abordaremos las formas en las que se fueron articulando las reflexiones de la interseccionalidad entre género y clase, nacidas de las experiencias sistemáticas de encuentro con la mujer pobladora y campesina, que estructuraron un sentido femenino de lo popular y que organizó uno de los ejes de los contenidos de la democracia por venir.

\section{La red organizacional y textual: Proposiciones, Apuntes para el Trabajo Social y Flacso.}

La Revista Proposiciones comenzó a publicarse a comienzos de la década de los 80 y constituyó un espacio de sociabilidad político intelectual reconocido como parte de la renovación socialista. Nació como un proyecto de la ONG Sur Profesionales, con el objetivo de disponer de un espacio de discusión y debate para recomponer la crisis ideológica que azotaba a la izquierda, posterior al golpe de Estado, y que buscaba tomar distancia de las formas dogmáticas que habían dominado la experiencia intelectual de la izquierda de los años 60-70. Como lo indicaba su título, la revista buscaba "proponer" formas reflexivas que, alejadas del marxismo ortodoxo, resituaran a la izquierda en una perspectiva democrática, con nuevas claves analíticas que permitieran comprender el fracaso que había 
significado el Golpe de Estado y las transformaciones profundas que instalaba la dictadura a través de su proyecto neoliberal (Moyano "La Revista").

Entre 1980 y 1989, Proposiciones publicó un total de 161 artículos en 17 números. De ellos 21 fueron escritos por mujeres -economistas y trabajadoras sociales, principalmente-, en las que abordaron problemáticas derivadas de la experiencia de investigación con mujeres pobladoras. Con técnicas de corte cualitativo como historias de vida, educación popular y talleres, revalidaron las prácticas de la intervención acción, resignificando la experiencia de generación de conocimiento. Concentradas en la reflexión sobre las particularidades de la experiencia femenina, intentaron aportar con un análisis que combinara la clase y el género, proponiendo incorporar a las discusiones socialistas, categorías analíticas que no habían formado parte de los corpus tradicionales de la izquierda.

La gran mayoría de las intelectuales que escribieron en Proposiciones, formaron parte de otro espacio de sociabilidad intelectual denominado Colectivo de Trabajo Social. Formado en los inicios de la misma década, trabajadoras sociales que estudiaron en la Universidad Católica se dieron a la labor inconclusa de renovar la teoría que fundamentaba el trabajo social y que apenas había alcanzado a ser esbozada en los inicios de los años 70. Así, vinculadas a una red más amplia que alojaba el Centro Latinoamericano de Trabajo Social, CELATS, (con sede central en Perú), estas mujeres organizaron la iniciativa de publicar la revista Apuntes para el trabajo social, que entre 1981 y 1990 editó 17 números ininterrumpidos y en los que se intentó sistematizar los debates respecto del Trabajo Social Alternativo, corriente que buscaba redefinir la práctica intelectual en el equilibro entre la generación de saber científico y la promoción de actividades de educación popular, para potenciar la transformación colectiva y consciente de los sujetos subalternos.

Esta revista, que a diferencia de Proposiciones, tuvo un carácter marcadamente académico profesionalizante, buscó convertirse en un referente para los trabajadores sociales, en términos estricto y amplio - es decir, como asistentes sociales y como educadores populares-, además de consolidar un espacio de sociabilidad intelectual donde el habla femenina tomaba la textura histórica de una 
experiencia de intervención acción, asociada a la disciplina desde su creación en las postrimerías de 1920 (Moyano y Pacheco).

Varias de las autoras de este colectivo también participaron del espacio de unos de los centros académicos más relevantes de los años 80, la Facultad de Ciencias Sociales (FLACSO). Dicha institución creada con anterioridad al Golpe de Estado, asociada al proceso de consolidación de las ciencias sociales en América Latina, se constituyó en un hábitat fecundo para el conjunto de intelectuales que permanecieron en el insilio nacional durante la larga dictadura militar chilena. A diferencia de las otras organizaciones, Flacso no promocionó iniciativas de publicaciones periódicas en modelo de revistas, sino que organizó su producción en documentos de trabajo y en libros, cuando estos fueron permitidos por los organismos gubernamentales de facto. Destacamos aquí la importante labor que desarrolló Teresa Valdés, como principal voz que, desde el feminismo, reunió los trabajos de otras intelectuales vinculadas a la reflexión sobre lo popular y la experiencia femenina de las pobladoras. Cabe precisar que Flacso tuvo entre sus filas a otras importantes figuras del feminismo socialista, entre las que resalta la figura de Julieta Kirwood, constituyéndose así en un espacio de resonancia particular del debate político, tanto para mujeres y hombres de la elite socialista y demócrata cristiana nacional, como en un ámbito más transnacional.

El modelo de documentos de trabajo o de material de discusión, que fue el predominante en esta institución para todos los años que contempla este artículo, permite no sólo rastrear formas particulares de producción intelectual en las ciencias sociales chilenas, sino también revisitar los espacios de reunión que dieron soportes a las redes intelectuales de la renovación socialista. Muchos de los trabajos que adoptaron el formato antes indicado fueron objeto de presentación en coloquios y seminarios nacionales o internacionales, permitiendo restituir las huellas de las inscripciones en las que se situaron los textos, los debates más generales de su circulación y, por cierto, la amplitud espacial de una textualidad que subvertía las normativas de la censura vigente. Por último, varios de estos documentos de trabajo fueron referenciados en las revistas Proposiciones y Apuntes, como parte del corpus 
teórico y empírico que sustentaba los hallazgos y afirmaciones de las y los intelectuales, dando cuenta de que pese a la declaración de ser un "texto" no acabado para una circulación formal o institucional, fue usado como tal por los productores de saberes en los años ochenta. Entre 1980 y 1989, Flacso publicó un total de 275 documentos de trabajo, de los cuales 47 tuvieron por objeto de estudio al mundo popular, los pobladores y más específicamente a la mujer. Estos últimos contaron además con el financiamiento de PISPAL, la CEPAL y CLACSO, además de haber sido objeto de presentación en Congresos y reuniones realizadas en Buenos Aires, Boston y Canadá.

\section{Los años de redescubrimiento de la mujer popular, 1982-1987.}

La cesantía que generó la crisis de 1982 y una serie de temporales que azotaron Santiago en ese mismo año, afectaron de forma intensa al mundo popular. Junto a la represión política existente y el deterioro de los hábitats poblacionales, se comenzó a figurar la idea de una crisis y de una emergencia social. Intelectuales vinculadas a las distintas Vicarías Zonales y ONG realizaron trabajos de intervención social que buscaban colaborar en la resolución de los problemas cotidianos, pero también dotar de una conceptualización "politizadora" que resignificara las redes de sociabilidad popular. Así, junto con la aparición de programas como "Comprando Juntos", liderado por el Programa de la Economía del Trabajo (PET), más otros numerosos talleres financiados por ONGs, fueron ocupando el espacio poblacional para colaborar y también para re-politizar los lazos de asociatividad local. Surge aquí el concepto de "Organizaciones Económicas Populares" (OEP) que, sistematizado por Luis Razeto, economista del PET, y Clarisa Hardy, socióloga de la misma institución; se estructuró como categoría analítica que reunía las prácticas de asociatividad popular proactiva para enfrentar los efectos de la dura crisis económica: el hambre y la cesantía. Ollas comunes, comedores populares, fueron comprendidos como experiencias de una memoria 
popular que cobraba nuevos bríos en el contexto dictatorial, que reestructuraba las dinámicas políticas y que potenciaba al sujeto como agente activo de su propio entorno, disputando las lógicas del modelo neoliberal cuya crisis se experimentaba agudamente en las poblaciones de todo Chile.

Ya no era simplemente el poblador, sino que el poblador organizado. Sin embargo, algo comenzó a aparecer con fuerza en los textos de estos años. Ese concepto de poblador invisibilizaba la experiencia femenina, que se trastocaba profundamente con los mismos efectos de la crisis. Así, el encuentro con la mujer popular, en las ollas comunes, en las guarderías infantiles comunitarias, en el "comprando juntos", habilitó a las intelectuales que hacían intervención social para reflexionar sobre las particularidades de la experiencia femenina popular. La crisis de 1982 estaba trastocando los cimientos de la familia popular y quien experimentaba con mayor fuerza estos cambios era precisamente la mujer, encapsulada en una serie de estereotipos en sus roles de madre, dueña de casa y esposa, que se redefinían en el marco de la sociedad patriarcal y las reorientaciones neoliberales.

Según Teresa Valdés, entre 1980 y 1989 el 18\% de los productos de investigación producidos por mujeres intelectuales se concentraron en organizaciones y actores sociales, seguido por un 11,2\% de temas referidos a economía y trabajo, un 9,7\% en área de política, sistemas y procesos políticos; y un 20,6\% referidos a identidad mujer, familia y pareja, sexualidad y violencia contra la mujer. Si sumamos aquellos que desagregadamente la autora dispone como organizaciones y actores sociales, movimientos sociales, mujeres y población, el número sube a cerca del $30 \%$ (90).

En Proposiciones No 9 de julio de 1983, Paulina Saball y Andrea Rodó, también editoras de la revista Apuntes para el trabajo social, formalizaron el conjunto de los dilemas de la mujer popular. Este artículo formó parte de los resultados emanados del "Proyecto Mujeres Pobladoras", dependiente de SUR y más en particular, de los resultados de los "Talleres de Formación de la mujer pobladora". En este texto, aparece no sólo una descripción de la materialidad de lo 
popular, sino que también colabora a una importante diferenciación entre mujeres de hogares obreros y mujeres que viven en campamentos. La distinción obedece a la consignación de una heterogeneidad mayor en lo popular, basada en la importancia de la materialidad de la experiencia societal. La pobreza en casa de obreros y en los campamentos se configura, se siente y se expresa de forma distinta, planteaban las autoras. La comprensión de una diversa constitución de la experiencia femenina popular será uno de los temas más relevantes en estos años: "La mujer aquí es fuerte, alegre, agresiva ("peleadora”), celosa y decidida. No es la mujer con vocación de dueña de casa y esposa" (50), concluyen Saball y Rodó.

El deterioro de la sociedad del trabajo reconfiguró las identidades colectivas, sobre todo en aquellos sectores sociales donde el trabajo habría sido el centro de las trayectorias de vida. Sin embargo, en aquel mundo popular donde el trabajo salarial fue inestable, precario y casi inexistente, las identidades tenían otra fuerza constitutiva. Fue en este espacio de las mujeres populares, donde las autoras centraron sus esperanzas políticas, ya que aquí era posible la rebeldía. Indicaron en Proposiciones $\mathrm{n}^{\circ}$ 9:

Al parecer muchas mujeres de este grupo social han empezado a conocer la rebeldía. Ahora no realizan su trabajo en forma aislada y esporádica, como empleadas domésticas o lavanderas. Son cuadrillas de mujeres, vecinas, amigas, trabajando en un mismo lugar bajo el PEM (Programa de Empleo Mínimo), con sus maridos cesantes parados en las esquinas, jugando a la pelota o alcoholizados y los niños solos. La mujer del campamento toma conciencia que, para bien o para mal, ella es el pilar para que todos puedan seguir sobreviviendo. (54)

Este análisis formó parte de los debates más amplios que estaba revisitando la izquierda, respecto de las categorías analíticas que formaban parte de los corpus teóricos que habían usado para generar conocimiento y transformar la realidad. Detrás de esta reflexión, no sólo se encuentra el rescate de la experiencia 
poblacional, sino que por sobre todo una redefinición de lo popular, para llenar de nuevos sentidos a la categoría de clase. De allí que el descubrimiento de una mujer popular heterogénea sea tan relevante, porque en la misma población o en espacios de pobreza urbana, se constituirían experiencias de significación de las identidades sociales diferenciadas. El deterioro de los vínculos salariales, la crisis económica, el desempleo y la carestía habrían sido experimentados de forma distinta en las mujeres populares. En unas se construía la resignación y la impotencia, en otras la rebeldía.

Lo popular, por tanto, erosionó el antiguo concepto de clase de corte marxista estructural, para incorporar aspectos referidos a las experiencias significativas de los sujetos. Con ello se tensionó la mecánica relación entre estructura y superestructura y se obligó a definir las categorías de ideología, de partidos políticos y movimientos sociales. El debate introducido sobre la heterogeneidad de la mujer popular puede ser leído en esta clave más amplia de los procesos de renovación de la izquierda y desde allí aparece jugando un rol relevante que, cruzado con el feminismo, posibilitará la construcción de una "demanda" de las mujeres respecto de la democracia y la democratización por venir.

La necesidad de dar visibilidad y relevancia a las mujeres populares y poblacionales cruzó de forma sistemática los textos que constituyen la revista Apuntes para el Trabajo Social. En dicha revista, los temas claves "que preocuparon a las intelectuales asociadas al Colectivo de Trabajo Social, era dar cuenta de la condición de explotación social, subordinación patriarcal y política que vivían las mujeres en las poblaciones" (Moyano y Pacheco 24). El rescate de las experiencias derivadas de la promoción de actividades de subsistencia colectiva, en conjunto con las reflexiones emanadas de los talleres de sexualidad y vida afectiva, configuraron un corpus de conocimiento sobre las mujeres populares que impregnó el debate sobre la política y la democracia. Las jornadas de protesta popular iniciadas en mayo de 1983, les entregaron a estas reflexiones un contexto material de justificación y fuerza renovadora. El mundo poblacional se convierte en objeto de estudio y de debate político, como es posible distinguir en la Revista 
Proposiciones $\mathrm{N}^{\circ} 13$ de 1987. En la editorial del número se enfatiza la problemática de la necesidad de considerar a este nuevo (aunque viejo) actor social subalterno, excluido y explotado, como parte de los debates políticos de una futura democracia. En la publicación se resaltó además que:

[...] en la vida cotidiana de las mujeres pobladoras - materia de otro de nuestros artículos - se refleja con singular dureza el conjunto de las características del mundo poblacional; ellas encarnan en su cuerpo los dolores, las frustraciones, las alegrías y los pocos elementos de liberación a su alcance, canalizados a través de una maternidad idealizada que, finalmente, los desvirtúa. Porque ya en el terreno de lo cotidiano, el trabajo es esclavitud, es la pesada carga de la sobrevivencia; el sexo posee la ambigüedad del reconocimiento y de la atracción, pero se ve cercenado por las condiciones materiales de hacinamiento y por una cultura autoritaria en el terreno familiar, afectivo y físico. (8-9)

De allí que "la indagación en las representaciones colectivas que las mujeres tienen de sus cuerpos conduce a descubrir la matriz del sometimiento y de una posible liberación" (9). Por ello, los estudios conducidos en esta área debían propender a la transformación cultural, "la democratización de la reproducción social y de los espacios personales" que devienen en tareas urgentes (Valdés, "Ser mujer" 5), según las autoras feministas.

Estos debates que transitan en las páginas de las revistas que contienen escrituras feministas, fueron factor y producto de un auge en la movilización social. Para Teresa Valdés fue en estos años donde "lo específico femenino comenzó a abrirse paso en un contexto en que la apertura política aparecía inminente, adquiriendo legitimidad en la oposición democrática" (Valdés, "El movimiento" 40). Son los años de la creación del Centro de Estudios de la Mujer, del Centro de profesionales DOMOS, del taller de la Mujer del Instituto para el Nuevo Chile, cuyos estudios se difundían en redes de comunicación sostenidas por FEMPRES, 
ILET e Isis Internacional. Entre 1983 y 1987 también se había reconstituido el MEMCH; habían surgido nuevas organizaciones como la Unión Chilena de Mujeres, la Unión de Mujeres Rosario Ortiz vinculadas a sectores del partido socialista, la Federación de Mujeres Socialistas del Partido Socialista, la Coordinadora de Mujeres Humanistas, en conjunto con la emergencia de coordinadoras como el Movimiento de Mujeres por el Socialismo, Mujeres por la Vida, Movimiento Feminista la Morada, el Departamento de la Coordinadora Nacional Sindical y el Departamento Femenino de la Comisión Nacional Campesina. La incorporación de una integrante de Mujeres por la Vida, en representación del movimiento de mujeres, para la Asamblea de la Civilidad de 1986, también sería un punto que daba cuenta de la potencia de las hablas y accionar feministas (Valdés "El movimiento" 44).

Y aunque la diversidad de actores de este período no nos permite confirmar una homogeneidad feminista, lo cierto es que las revistas que hemos abordado textualizan parte de los ejes de este debate político y teórico, convirtiéndose en huella de las trasformaciones en la generación del conocimiento social y de la lucha por la emancipación de las mujeres, en clave de interseccionalidad entre género y clase. Así, "desarrollo de conocimiento" y "desarrollo de movimiento social” se mantuvieron en coexistencia y se impulsaron mutuamente. Aun cuando aquello potenció la emergencia de las hablas feministas, también es cierto que tensionó el “desarrollo del pensamiento teórico y de la investigación con la construcción de un ser social colectivo- el movimiento de mujeres- destinado a promover la transformación de la sociedad y la cultura" (Valdés, "El movimiento" 54). Dado que el tiempo de la acción era un tiempo urgente, rápido, ansioso, presionó sobre el tiempo de la reflexión y acumulación de conocimientos. "Cuando una misma persona es activista e investigadora, se fusionan los intereses influyendo uno sobre otro [...] polarizando los marcos teóricos y las formas de investigar" que incidirán en cierta marginación académica, por lo que es posible distinguir que esta fue una etapa donde la generación de conocimiento sobre la experiencia femenina estuvo 
marcada por la ideología, además de cierta debilidad en el "rigor teórico y metodológico" (54).

Mucho del trabajo de estas mujeres intelectuales, estuvo vinculado con experiencias de investigación-acción y educación popular. La metodología de taller fue la más usada para este tipo de actividades, marca que quedó expresada en sus textos y también en el mundo popular, a través de la promoción de boletines, entendidos como órganos de expresión de condiciones de vida, de subjetivación y politización de lo cotidiano.

Boletines de Mujeres entre 1980-1989.

\begin{tabular}{ll}
\hline \multicolumn{1}{c}{ ÓRGANO DE DIFUSIÓN } & \multicolumn{1}{c}{ GRUPO U ORGANIZACIÓN QUE LO ELABORA } \\
Y nosotras qué & Taller de Mujeres Reflexión de la parroquia Santa Cristina. \\
\hline Mujeres en la lucha & Elaborado por Presas Políticas de Nueva Imperial \\
Oye vecina & Comité de Defensa de los Derechos de la Mujer (CODEM), Maipú. \\
Presencia de mujer & Departamento Femenino del Movimiento Sindical Unitario (MSU) \\
Hojita & Comisión de Derechos de la Mujer \\
Guacolda & Acción Femenina \\
\hline Mujer, voz y pueblo & De mujeres pobladoras de la población Herminia \\
Nuestro despertar & De los Talleres Solidarios José María Caro \\
Ormiga & Unión de Mujeres de Atacama (UDEMA) \\
Palabra de mujer & Grupo Las Domitilas \\
\hline Palomita & Agrupación de Mujeres Democráticas \\
Remolino & Movimiento Pro Emancipación de la Mujer Chilena (MEMCH-83) \\
La Boletina & Frente de Liberación Femenina de Chile \\
Testimonio & Ediciones feministas Centro Mujer \\
Nos/otras & Sindicato Interempresas de Trabajadoras de Casa Particular \\
Boletín Sintracap & (SINTRACAP). \\
\hline Vamos mujer & Comité de Defensa de los Derechos de la Mujer (CODEM) \\
\hline Furia & Federación de Mujeres Socialistas \\
Boletín del Círculo & Círculo de Estudios de la Mujer \\
Boletín Bimestral La Morada & Corporación Casa de la Mujer La Morada \\
Tu voz Mujer & Comité de Defensa de los Derechos de la Mujer (CODEM), \\
La Cigarra & Concepción. \\
Boletín Ridem & Comité Pro Unidad de la Mujer \\
\hline & Red de Información y Difusión de la Mujer (RIDEM) \\
\hline
\end{tabular}

1. Elaboración propia en base a catastro realizado en el marco del proyecto Fondecyt 1150049. 
Con todo, estos años estuvieron marcados por los debates sobre lo social popular y desde el feminismo socialista. Lo popular venía a visibilizar la estrechez de la categoría de clase, incorporando la perspectiva de género. Por ello, descubrir y recrear la experiencia femenina de lo popular se convirtió en uno de los ejes centrales que las mujeres que formaron parte de las revistas Apuntes, Proposiciones y de Flacso, rehabilitaron como parte del debate académico y también político.

\section{De lo popular a lo democrático.}

El itinerario del debate feminista no se agota por cierto en estas revistas, su complejidad nos aparece constantemente hasta nuestra contemporaneidad. Sin embargo, es posible distinguir que hacia el año 1987 las mujeres intelectuales que escriben en ellas van resaltando con mayor nitidez la necesidad de referir a las prácticas políticas y la democracia porvenir. Del rescate de la experiencia sociopopular femenina se deriva con mucha rapidez a la mujer en democracia.

Así, considerando el fracaso de la vía armada para derrocar a la Dictadura, la disminución de la intensidad y periodicidad de las jornadas de protesta popular y el fracaso de la Asamblea de la Civilidad en 1986, el horizonte plebiscitario se hizo bastante más cercano y urgente. El acuerdo de las elites partidarias socialistas y demócratas cristianas de respetar el itinerario de la transición diseñado en la Constitución de 1980 por la propia Dictadura militar reorientó los ritmos del debate y la preocupación por la política, y lo democrático se tomó con fuerza el centro de los debates de las revistas políticas y las escrituras feministas.

Lo central ya no pasaba por la narración de las experiencias de las mujeres populares, sino que la disputa sobre cómo ingresaba la mujer en la política y, sobre todo, cómo las demandas feministas -orientadas a erosionar las dinámicas patriarcales- podían ser contenidos de una futura democracia. Las experiencias de asociatividad popular fueron releídas en el marco de la democratización, es decir, como expresiones previas de sociabilidad popular que podían "re-significar" las 
experiencias democráticas de antaño y las futuras. Sin embargo, el objetivo de articular los ejes entre clase y género pasó a un segundo plano. La urgencia de los tiempos obligaba a repensar la democracia y con ello se postergó la presencia de lo popular.

En conjunto con lo anterior es necesario resaltar que las autoras de estas revistas formaron parte activa de la Concertación de Mujeres por la Democracia, nacida en 1988 como instancia autónoma -pero en diálogo- con la Concertación de Partidos por la Democracia. La creación de esta orgánica vino a dar cuenta:

[...] que el movimiento de mujeres había logrado que sus problemas se socializaran, formaran parte del discurso público, adquiriendo un rol relevante en cuanto actor del proceso de la sociedad chilena. Por otra parte, revelan una etapa particular en la vida del movimiento por cuanto, tras largos años de acción, elaboración y debate, se había consolidado una capa de profesionales que buscaba alternativas de modificación de la condición femenina desde sus especialidades. Se agrega a este desarrollo un nuevo factor de gran relevancia y es la articulación con mujeres de partidos políticos. En conjunto diseñan una estrategia para la inserción de la agenda del movimiento en el futuro gobierno democrático. (Valdés "El movimiento" 156)

La ruta en este sentido estuvo plagada de varias tensiones que quedaron enunciadas en las páginas de estas revistas. Por un lado, el debate por la democracia obligó de repensar la relación entre movimiento y partido, la autonomía y la doble militancia. Por el otro, implicó reflexionar sobre la política en clave femenina o en cómo feminizar las prácticas políticas, para configurar una nueva política.

En el boletín "Nosotras" de 1984 se expresaba que:

[...] la autonomía del movimiento hace alusión a la necesidad de un movimiento independiente, tanto a nivel organizativo como de las 
reivindicaciones específicas de la mujer, garantizando que éstas no se diluyan en las múltiples contradicciones sociales y políticas ni en los intereses de determinados grupos, clases o instituciones; hace referencia igualmente a la necesidad de tener un espacio de confrontación directa entre mujeres y, a partir de nuestros intereses, con el resto de la sociedad, sin mediatización. (separata)

La apuesta por la autonomía era parte de la necesidad de visibilizar que la “cuestión de la mujer" no podía quedar sólo en una enunciación, sino que debía politizarse desde las propias mujeres. Ad portas del plebiscito, se argumentaba la prioridad de mantener los temas en la agenda y no abandonar la propuesta emanada del $1^{\circ}$ Encuentro Feminista Latinoamericano y del Caribe, realizado en Bogotá en 1981, que interpelaba a que "las organizaciones feministas debían tener autonomía orgánica aunque los objetivos no sean autónomos de los demás sectores históricamente revolucionarios, sino que estén inter-relacionados con los intereses de la clase obrera por la supresión de la explotación clasista y con los de todo el pueblo por la derrota de la dominación imperialista" ${ }^{2}$.

Para las mujeres intelectuales de las ciencias sociales, el horizonte democrático obligó a repensar las opciones de autonomía. Tal como expresó en 1989 Natacha Molina, para el movimiento de mujeres "manteniendo en lo fundamental un fuerte sentimiento defensivo autónomo que invita al reconocimiento de una identidad colectiva de género, se percibe que la tarea pendiente es ingresar al proceso político nacional con una propuesta (agenda común) que puede presionar en cada una de ellas por incorporar propuestas de cambio que tengan sentido para las mujeres" (34).

Paulina Saball y Andrea Rodó sintetizaron esta problemática en el № 15 de Proposiciones, enfatizando que:

\footnotetext{
${ }^{2}$ Boletín de Mujeres, Nº, 1982:33.
} 
Sin duda alguna, una de las banderas de las organizaciones femeninas que han alcanzado presencia pública, ha sido la redemocratización de las relaciones humanas. Las mujeres - en su discurso y en sus formas de manifestación política- se han planteado explícitamente contra el autoritarismo, el sectarismo y las orgánicas asfixiantes [...] El desafío está en poder darse formas orgánicas que estén fundadas en la democracia, en la autonomía y el respeto a la diversidad; en la incorporación de lo subjetivo en la toma de decisiones; pero que a la vez sean eficientes, permitan tener presencia de movimiento $\mathrm{y}$, principalmente, desarrollar vínculos y formas de agrupación que sean adecuadas a las necesidades y que no se transformen en "organización burocráticas y superestructurales". (200)

Detrás de dicha reflexión se encontraba una crítica a las formas tradicionales de la política, en particular de los partidos políticos, de allí que el conflicto referido a las dobles militancias y los espacios de participación político institucional fueran otro elemento de los debates que se introdujo en la transición a la democracia. Para Saball y Rodó, "suponer que los procesos de autoconciencia generan por si mismos mayores y mejores niveles de acción colectiva, nos parece equivocado. Las prácticas de educación popular durante este período han puesto el énfasis en toma de conciencia, y poco han incidido en el desarrollo de la capacidad de gestión y negociación de las mujeres respecto de sus problemas" (200)

Quedaban así enunciados los límites de la acción femenina en el campo de lo político y las múltiples tensiones con los partidos y los dilemas de la autonomía. Estos dilemas no lograron resolverse, fracturando agudamente al movimiento de mujeres hacia fines de la década de los años 80 .

No fue una coincidencia que el último número de la revista Apuntes para el Trabajo Social se publicara ad portas del primer gobierno electo en 1990, y que el tema de la gobernabilidad se tomara la agenda de los debates en Flacso y en Proposiciones. Se había cerrado una etapa. Paradójicamente, la democracia 
contrajo las disputas y esta oleada de investigación acción se diluyó en la agenda de las mujeres, muchas de las cuales pasaron a ocupar cargos relevantes en la administración democrática. Los sueños de un feminismo popular quedaron en suspenso. Hasta ahora.

\section{Bibliografía}

Arfurch, Leonor. El espacio biográfico. Dilemas de la subjetividad contemporánea. Buenos Aires: Fondo Cultura Económica, 2002.

Boletín de Mujeres, Nº9, 1982.

Boletín Nos/otras, $\mathrm{N}^{\mathrm{o}} 1,1984$, separata.

Molina, Natacha. "Propuestas políticas y orientaciones de cambio en la situación de la mujer”. En: Manuel Antonio Garretón (comp.), Propuestas políticas y demandas sociales. Vol. III, Santiago: FLACSO, 1989, p. 41.

Molina, Natacha y Serrano, Claudia. "Las mujeres chilenas frente a la política", Proposiciones $\mathrm{N}^{\mathrm{o}} 16$ (1988): 73-83.

Moyano, Cristina. "La Revista Proposiciones: espacio de sociabilidad intelectual y producción de saberes en el campo intelectual de la izquierda chilena durante los años 80”, Revista Austral de Ciencias Sociales, 32 (2017)

Moyano, Cristina y Pacheco, Valentina. "Revista Apuntes para el trabajo social: una mirada a las mujeres intelectuales de las ONG y la generación de conocimiento sobre lo femenino-popular en Chile, 1980-1989”. Revista História (Sao Paulo), vol. 37, 2018. 1-24.

Olea, Raquel en Eliana Largo Vera, Calles caminadas. Anverso y reverso, Santiago, Centro de Investigaciones Diego Barros Arana, Dirección de Bibliotecas, Archivos y Museos, 2014.

Revista Apuntes para el Trabajo Social, números 1 al 17, 1980-1989. 
Rodó, Andrea. "El cuerpo ausente", Proposiciones 13 (1987): 109-164

Rodó, Andrea y Saball, Paulina. “Talleres de formación para mujeres pobladoras", Proposiciones 15 (1987): 213-216.

Valdés, Teresa. "Ser mujer en sectores populares urbanos", Documento de Trabajo $\mathrm{N}^{\circ}$ 269, octubre de 1985. . "El movimiento social de mujeres. La producción de conocimiento sobre la condición de la mujer", Documento de trabajo, serie estudios sociales $\mathrm{N}^{\circ} 43$, marzo de 1993. 